\title{
Advertising in Social-Media as A New Approach in Mental Illness Campaign Among Youth
}

\author{
Norshahira Atikah Mohd Razak ${ }^{1}$ \& Mohd Nasiruddin Abdul Aziz ${ }^{2}$ \\ Faculty of Art and Design, Universiti Teknologi MARA Perak Branch, Seri Iskandar Campus, \\ 32610, Seri Iskandar, Perak, MALAYSIA \\ Authors' email: nrshhratikah@gmail.com¹ mohdn571@perak.uitm.edu.my ${ }^{2}$
}

Published: 7 September 2018

\begin{abstract}
Nowadays, the mass media have often aired unexpected news of death and suicide. Peoples that under depression did not want to seek help and people surrounding them did not know how to help them either. There have been numerous efforts by various parties to address this problem time by time. However, previous related study shows an increasing number of major depressive episodes from the year 2010 until 2018 among young adults. Psychologists believe that the numbers will continue to rise in 2020 if no changes were made. This research intends to educate people on how to treat friends or family members that have a depression through advertising by using social media platforms to gain awareness among youngsters about the bad effect of depression. Contextual analysis from the case study and a survey has been conducted. Data have been collected from interviews, newspapers, and published reports. This research found that the majority of the respondents did not know how to help people that have depression and they usually take a wrong step while helping them. The social campaign was expected to be the key driver for change in the public's attitudes and behaviour.
\end{abstract}

Keywords: Mental illness, depression, social media, youngsters, campaign

eISSN: 2550-214X (C) 2018. The Authors. Published for Idealogy Journal of Arts and Social Science by UiTM Press. This is an Open Access article distributed under the terms of the Creative Commons Attribution-NonCommercial-NoDerivatives License (http://creativecommons.org/licenses/by-nc-nd/4.0/), which permits non-commercial re-use, distribution, and reproduction in any medium, provided the original work is properly cited, and is not altered, transformed, or built upon in any way.

\section{INTRODUCTION}

Four out of every 10 Malaysians will fall victim to some form of mental health issue especially in depression in the course of their lives. Psychologists believe that the numbers will continue to rise in 2020 if we did not make a change. We have not heard much from the Government to address this issue. The silent from the authorities is disappointing.

Several factors contribute to a person's depression. Firstly, is genetics or offspring. Individuals with family members who have had a depressive disease have a higher risk of developing this disease than those who did not. Besides, individuals with anxiety or unerring personality are more likely to get this disease. Psychological trauma in childhood or physical trauma on the brain due to injury or drug abuse can be one of the factors of depression. Accidents in childhood have greater impact and lead to a higher risk for depression for an individual in their lives. For those who use drugs, the risk of developing a depressive disease increases as well as those who do not take drugs.

Social media has become a mainstay in our lives. Sites like Twitter, Tumblr, Instagram and Facebook have changed the way we communicate with others. Eight in ten Malaysians who are online use Facebook, the most popular social media platform with over a billion and a half users and growing.

Nearly two hours the average social media user spends on their chosen platforms each day. That number is expected to increase day by day as these sites continue to develop. The large amount of time we spend on social media nearly every day has led to more feelings of anxiety and depression. It has also reduced the amount of time we spend interacting with family, friends and our wider social circle (Pantic, 2014). Research into social media and how it impacts our lives and our mental health is still fairly new. But many studies have found an increase in mental health problems due to heavy social 
media use, especially among adolescents and young adults.

\section{LITERATURE REVIEW}

\subsection{Social Media Cause the Depression}

Luxton (2012) mentioned that use of multiple social media platforms like Facebook, Twitter and Instagram are more strongly associated and interact with depression and anxiety among youngsters than the total amount of time they spend on social media. They feel more depressed when they use social media rather than happy feelings.

The analysis from Pantic (2014) showed that people who report using seven to 10 social media platforms had more than four times the risk of depression and anxiety than their companion who use no more than two platforms every day, even after adjusting for the total time spent on social media overall still give the big impact to society. In 2014, Primack and his colleagues sampled 1,787 young adults, ages 19 through 32, using an established depression assessment tool and questionnaires to determine social media use. The questionnaires asked about the 11 most popular social media platforms at the time: Facebook, YouTube, Twitter, Google Plus, Instagram, Snapchat, Tumblr, Pinterest and Vine.

Based on report symptoms, participants using seven to 11 platforms have three times more likely to have higher depression than their companion who use zero to two platforms. Those who use the most frequent platforms have four times the high level of emergency symptoms of their friends who use the least platform. The researchers dominate other internal factors that can contribute and lead to depression and anxiety, including gender, race, relationship status, household income, education and amount of time spent on social media.

\subsection{Contribution of Advertising to Mental IIIness Campaign}

\subsubsection{The Power of Okay - See Me}

See Me is a Scottish national program to end stigma and mental health discrimination to identify the workplace as a commonplace where the challenges take place. To address this issue, it assigns YouGov a collection aimed at identifying the causes of such stigmatization and discrimination, measuring 1,165 Scottish workers on their attitude towards mental health. As advertising and media professionals, they have the power to shape the culture. It is up to them to describe the mental illness accurately and regularly. Advertisers need to say "I have experienced personality border" easy as saying "I have allergies."

The results show that $48 \%$ of people do not tell their employers about mental health concerns for fear of losing their jobs. Also, 55\% think that someone at their workplace who is dealing with mental health problems especially depression, may not reveal it for fear of being moved or approved for promotion. These findings underscore the importance of talking to combat this fear. This campaign has produced a video based on a poem that includes the need to get people talking and asking a colleague, 'Are you okay?'. It has been shown online and cinemas, and also supported by a radio campaign.

The campaign, which was launched in November 2015, with a view of the site, which increased by $42.8 \%$ as a result, pulling $73 \%$ of visitors to the new website and a $22 \%$ rate of return visitors.

See Me identify and deal with the fear factor in this matter by adopting the language of everyday people in the campaign. In particular, it challenges people to think about the normal rotation phrase, 'Are you okay?' Normalize important conversation about how someone, but reinforce its importance. 


\subsection{2 \#HereForYou - Instagram}

\#HereForYou campaign was launched in May 2017. It was intended to encourage and give support to the existing community of people on Instagram to always support one another and find the appropriate help to help these individuals, spreading their support wider.

Three Instagram users are talking about their past struggles with depression, eating disorders and suicidal thoughts in the one-minute campaign video features. \#HereForYou flagship hashtag is used for the campaigns to attract the society - which are commonly used by Instagram users with the use of the hashtag.

To recognize the platform to gather a community of people, Instagram is the best platform which has spawned a community of people who come together to share experiences, stories, seek and find the support that they may need.

It knocked on the very real needs by opening its role in connecting people with the help they need, eventually developing societies.

\subsection{3 \#IAmNotAshamed}

In 2014, a World Health Organisation mental health report released on the profile of Malaysia showed that there are only one psychiatrist and one psychologist available for 100,000 people in Malaysia who are not enough to help all the patient. The campaign aims to encourage and give support to people to talk about their condition and the problems that they have been facing and seek professional help. It has encouraged many people by coming out to speak and share about their conditions and problems with the experts.

\section{METHODOLOGY}

Quantitative research approach through a questionnaire survey has been conducted. This method is chosen to get the feedback directly and accurately. Several sections have been covered through the questionnaire distribution. Total of 100 respondents was involved in the survey, and the method of selecting the respondent is purposive random sampling: between 18 to 35 years old which is involve students and working people. All the collected data has been recorded and analysed in Microsoft Excel.

The survey distribution was held at public places and in UiTM Seri Iskandar, Perak. Both of the venues fulfil the requirements for the survey to be conducted. The survey has been conducted from 11.00 am until $7.00 \mathrm{pm}$. All of the information was gathered and calculated manually three times to obtain the result.

\section{FINDINGS}

Figure 1.1 shows 50 respondents out of 100 stated that they did not know about the social issue of male sexual abuse. This portrays $61.11 \%$ of school students respondent did not seem aware that such cases did happen around them. 


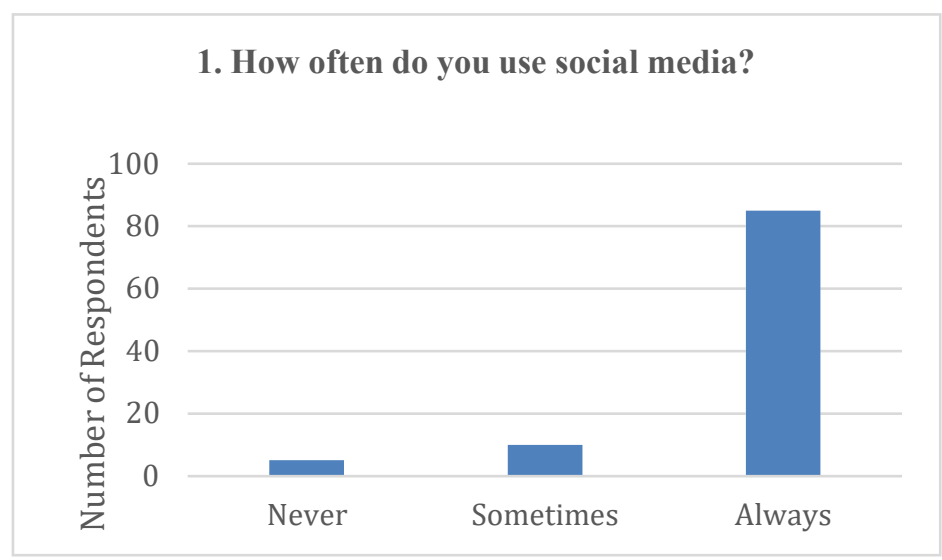

Figure 1.1 How often do you use social media?

Based on figure $1.1,83 \%$ per cent of respondents clearly stated that they are using media social as their daily life routine. None of them uses social media before. This is why the numbers in Malaysia are rising lately.

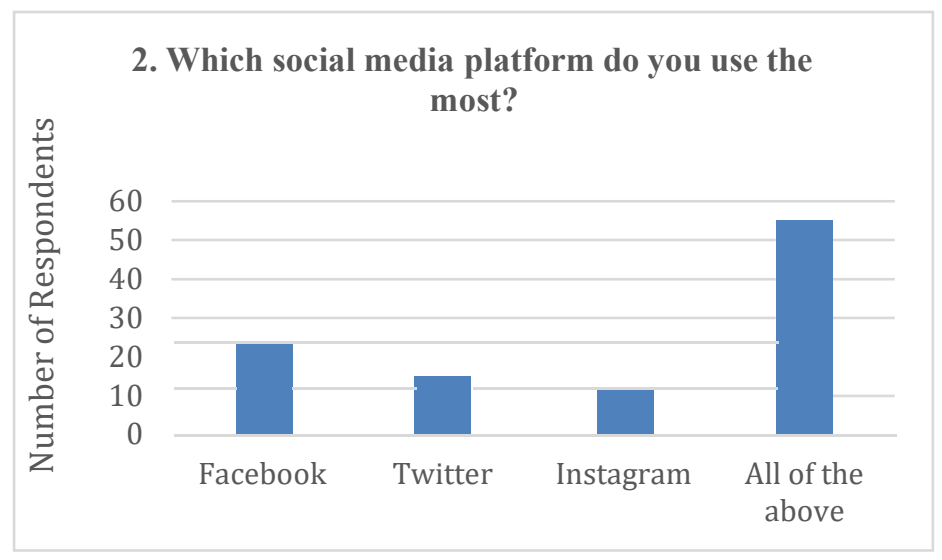

Figure 1.2 Which social media platform do you use the most?

Based on figure 1.3 below, the $55 \%$ of the respondents pick "all of the above", which means they probably use all of the social media to share their daily life routine including problems and personal life. The least of social media users is Instagram which is $10 \%$ from them. 


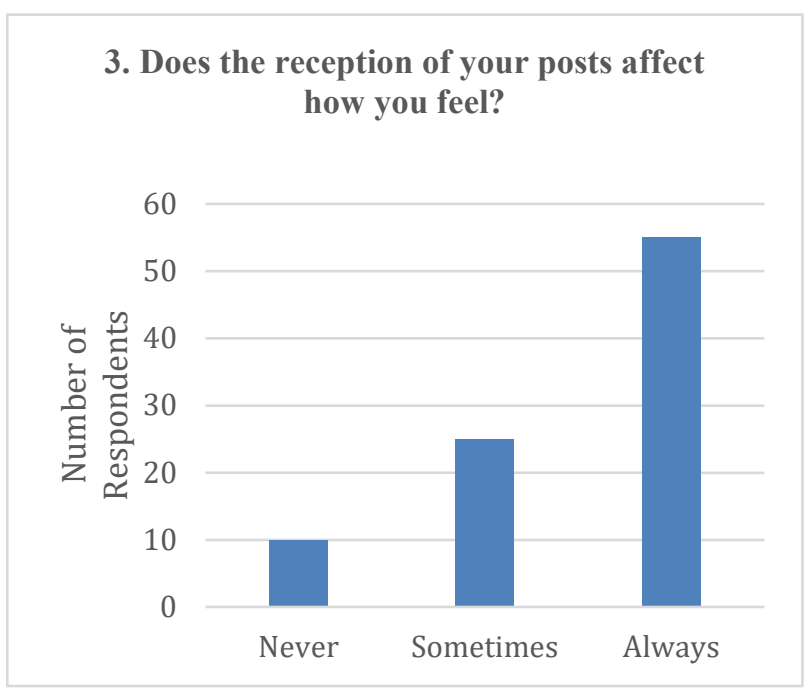

Figure 1.3 Does the reception of your posts affect how you feel?

While on figure 1.4, 55\% stated that the reception of their posts always affect how they feel. This shows that most of the posts from social media are from their feelings and current situations. $10 \%$ of them have never been affected by their feelings when they post something on social media.

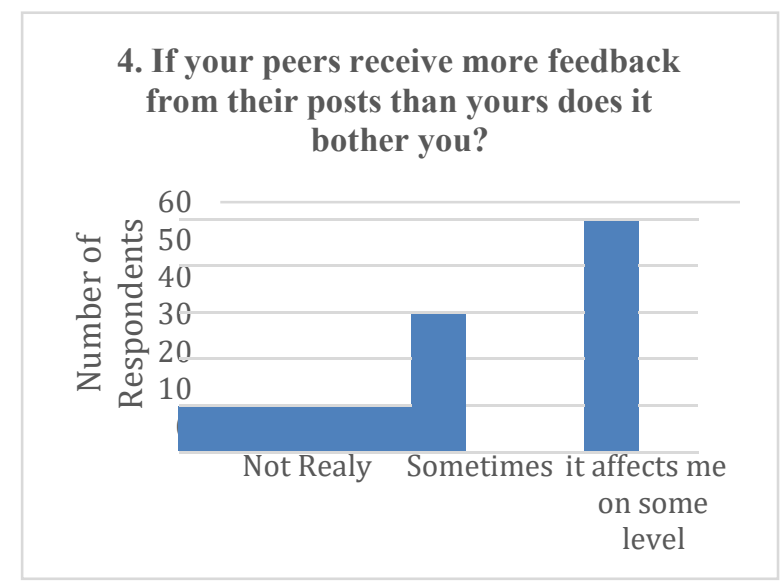

Figure 1.4 If your peers receive more feedback from their posts than yours does it bother you?

According to figure 1.5 below, $15 \%$ of respondent do not care about their peers if they are getting more feedback from their posts than them and it does not bother them at all. The $50 \%$ of the respondents have affected them on some level if they are getting less feedback than their peers. This somehow shows that the mentality of society is in a critical phase. They care about what people think of them than try to improve themselves. 


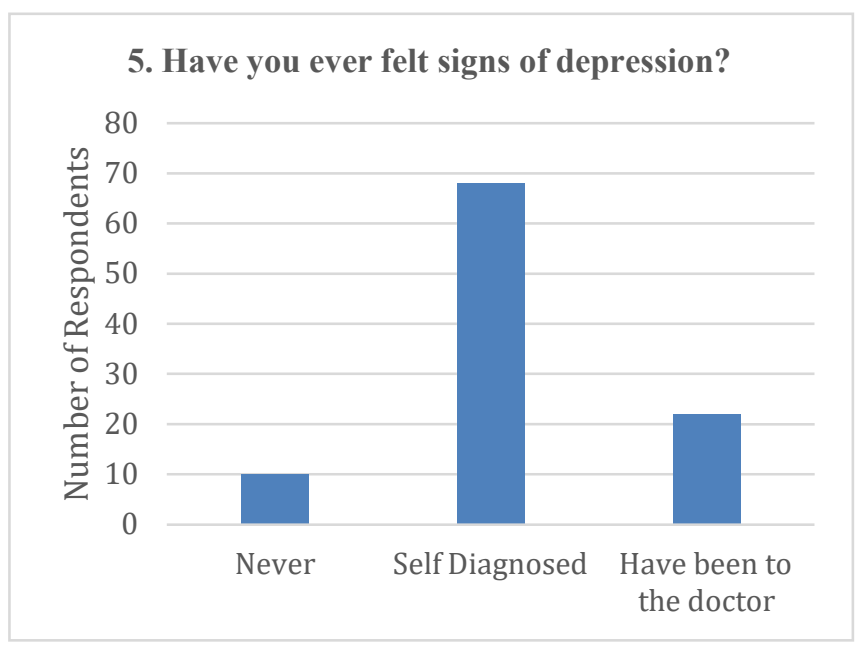

Figure 1.5 Have you ever felt signs of depression?

On figure $1.5,68 \%$ of the total of respondent have self-diagnosed with depression. $10 \%$ of them never felt signs of depression and $22 \%$ of them have been to the doctor for a monthly check-up. This shows that society or patient that have depression did not want to seek for help although they know that they have depression or mental illness. They are afraid that people will think they are crazy. This is one of the main problems with the society that has depression nowadays.

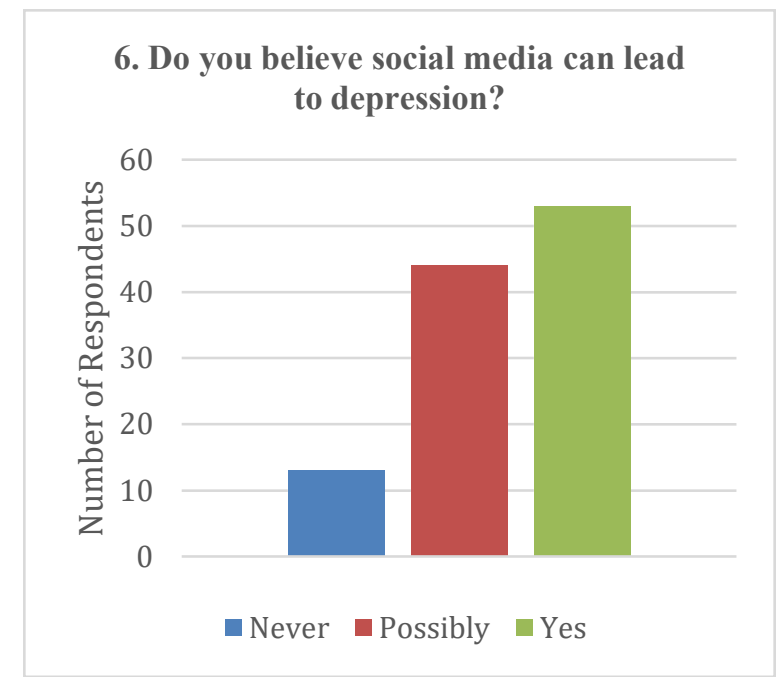

Figure 1.6 Have you ever felt signs of depression?

On figure $1.6,52 \%$ of the total of respondent had picked 'yes' when they answered the question. This proves that they agreed and believed that social media could lead to depression. This is based on their thought and experience of depression on social media. $43 \%$ of them picked because they are rarely feeling under depression. 


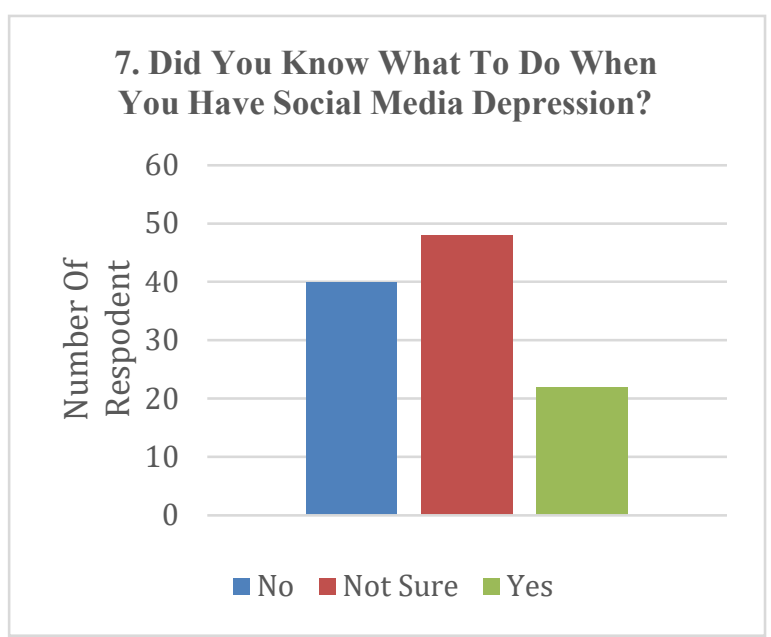

Figure 1.7 Did you know What To Do When You Have Social Media Depression?

On figure $1.7,52 \%$ of the total of respondent had picked 'yes' when they answered the question. This proves that they agreed and believed that social media could lead to depression.

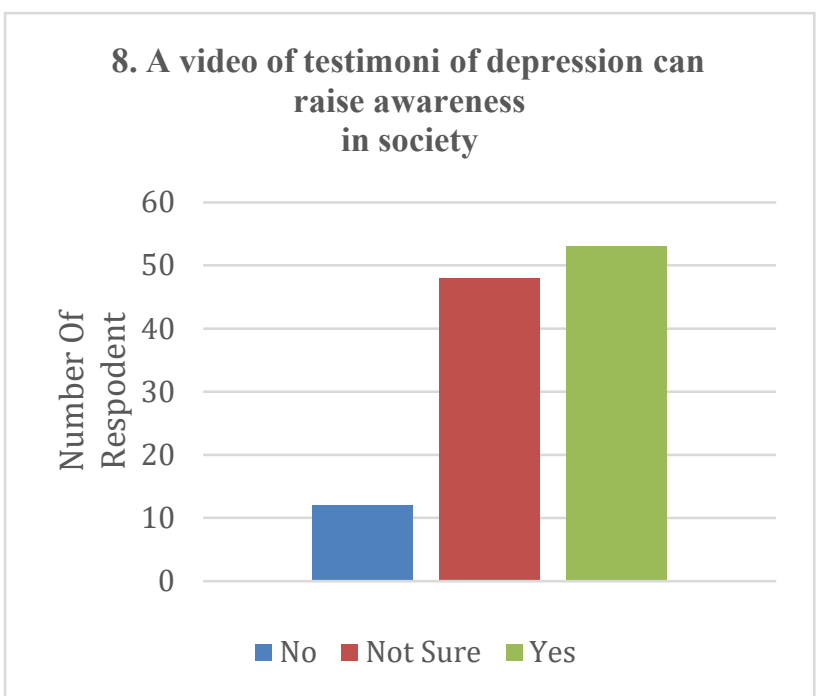

Figure 1.8 Did you know What To Do When You Have Social Media Depression?

While on figure $1.8,52 \%$ of the total of respondent had picked 'yes' when they answered the question. This proves that they believed a video of the testimony of depression could raise awareness in society. $48 \%$ said that they are not sure and $12 \%$ have picked 'no'. 


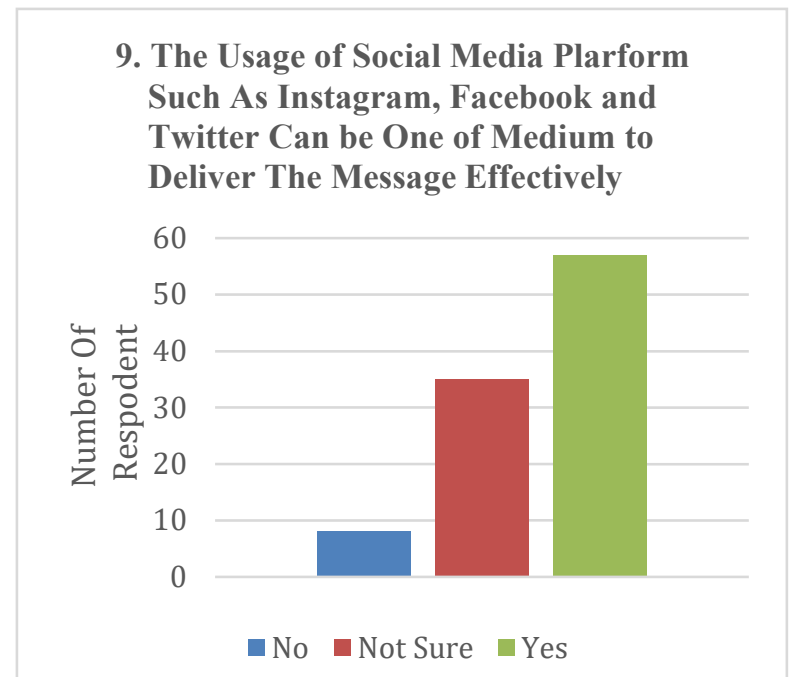

Figure 1.9 The Usage of Sociai Iiedia Piatform Such Âs instagram, Facebook and Twitter Can be One of Medium to Deliver The Message Effectively

On figure 1.9, 57\% of the total of respondent have picked 'yes' from the question given. This show that they agreed that social media platforms such as Instagram, Facebook and Twitter can be on of medium to deliver the message effectively among youngsters. This is because they use most of their time on social media. $8 \%$ from them said no, and $35 \%$ are not sure.

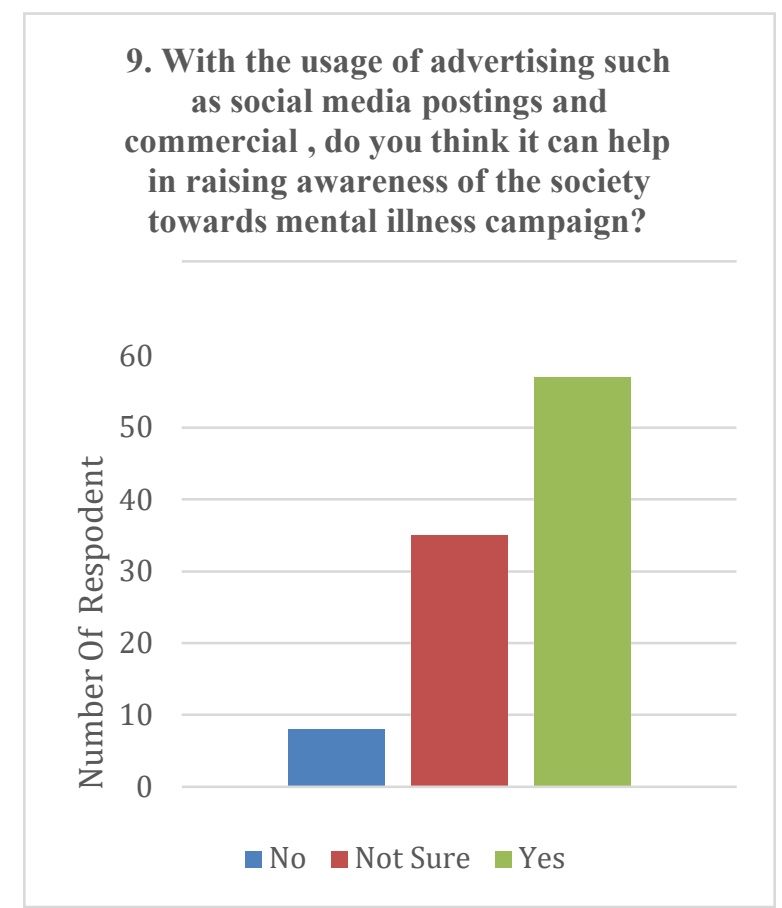

Figure 2.0 With the usage of advertising such as social media postings and commercial, do you think it can help in raising awareness of the society towards mental illness campaign?

On figure $2.0,57 \%$ of the respondent agreed and said yes that the usage of advertising such as social media postings and commercial, can help in raising awareness of the society towards mental illness campaign. They believed that advertising could help people dealing with depression by convincing them through postings and commercial. $8 \%$ of them are said no, and the rest are not sure that advertising can help to raise awareness or not. They believed only doctors could help them. But with advertising, it can be one of medium to help doctors treat the patient that have depression. 


\section{CONCLUSION}

Advertising does play a major role in raising and giving awareness to the society by conveying and delivering intended messages in social media. Mental health has always been a very taboo topic in Asia, surprisingly much more so than sex. Asians have generally been taught to be conservative from youth. It is hard to say whether or not mental health is even a constant idea within the largest continent in the world. But because of modern technology, thanks to the large involvement of television and magazines, and more often than not, the whimsical world of the Internet. One can say that the intention for change is there.

Based on these research findings, it can be described that advertising does play a major role in increasing and giving awareness to the public about the mental health issue. This due to the society is lack of knowledge and easy to have depression when they do know how to overcome it.

Depression in social media can cause emotional and mental problems, and it can lead to overuse and even addiction. Knowing how the social media use affects the mental health is the first step in changing the way in using it. Social media should be used healthily to restore the quality of life directly.

\section{REFERENCES}

Cash, H., Rae, C. D., Steel, A. H., \& Winkler, A. (2012). Internet Addiction: A Brief Summary of Research and Practice. Current Psychiatry Reviews, 8(4), 202-298. Retrieved from http://www.ncbi.nlm.nih.gov/pmc/articles/PMC3480687/

Everette James, JD, MBA., director of Pitt's Health Policy Institute, is senior author of the research publication. Additional authors are Ariel Shensa, MA, Erica Barrett, Jaime E. Sidani, PhD, MPH, and Jason Colditz, MEd.The research was supported by the National Institutes of Health grant number R01-CA140150.

Greenwood, S., Perrin, A., \& Duggan, M. (2016, November 11). Social Media Update 2016. Retrieved from http://www.pewinternet.org/2016/11/11/social- media-update-2016/

Hurst, N. (2015, February 3). If Facebook Causes Envy, Depression Could Follow. Retrieved from http://munews.missouri.edu/news-releases/2015/0203- iffacebook-use-causes-envy-depressioncould-follow/

Luxton, D. D., June, J. D., \& Fairall, J. M. (2012). Social Media and Suicide: A Public Health Perspective. American Journal of Public Health, 102(Suppl 2), S195- S200. Retrieved from http://www.ncbi.nlm.nih.gov/pmc/articles/PMC3477910/

Pantic, I. (2014, October 1). Online Social Networking and Mental Health. Cyberpsychology, Behavior, and Social Networking, 17(10), 652-657. Retrieved from http://www.ncbi.nlm.nih.gov/pme/articles/PMC4183915/

Social Media Use Associated with Depression Among U.S. Young Adults. (2016, March 22). Retrieved from http://www.upmc.com/media/NewsReleases/2016/ Pages/lin-primack-sm-depression.aspx

Widen, G., Lindstrom, J., Brannback, M., Huvila, I., \& Nystrom, A. (2015). Mixed Emotions in Active Social Media Use - Fun and Convenient or Shameful and Embarrassing? Retrieved from https://www.ideals.illinois.edu/bitstream/ handle/2142/73460/34_ready.pdf?sequence $=2$ 
Idealogy Journal of Arts and Social Science

Vol. 3 No. 2, 2018 